\title{
Scaling and isospin effects in quasielastic lepton-nucleus scattering in the relativistic mean field approach
}

\author{
J.A. Caballero ${ }^{\text {a,* }}$, J.E. Amaro ${ }^{\text {b }}$, M.B. Barbaro ${ }^{\text {c }}$, T.W. Donnelly ${ }^{\text {d }}$, J.M. Udías ${ }^{\text {e }}$ \\ ${ }^{a}$ Departamento de Física Atómica, Molecular y Nuclear, Universidad de Sevilla, 41080 Sevilla, Spain \\ ${ }^{\mathrm{b}}$ Departamento de Física Atómica, Molecular y Nuclear, Universidad de Granada, 18071 Granada, Spain \\ ${ }^{\mathrm{c}}$ Dipartimento di Fisica Teorica, Università di Torino and INFN, Sezione di Torino, Via P. Giuria 1, 10125 Torino, Italy \\ ${ }^{\mathrm{d}}$ Center for Theoretical Physics, Laboratory for Nuclear Science Department of Physics, Massachusetts Institute of Technology, Cambridge, MA 02139, USA \\ e Departamento de Física Atómica, Molecular y Nuclear, Universidad Complutense de Madrid, 28040 Madrid, Spain
}

Received 10 May 2007; accepted 1 August 2007

Available online 14 August 2007

Editor: W. Haxton

\begin{abstract}
The role of isospin in quasielastic electron scattering and charge-changing neutrino reactions is investigated in the relativistic impulse approximation. We analyze proton and neutron scaling functions making use of various theoretical descriptions for the final-state interactions, focusing on the effects introduced by the presence of strong scalar and vector terms in the relativistic mean field approach. An explanation for the differences observed in the scaling functions evaluated from $\left(e, e^{\prime}\right)$ and $(v, \mu)$ reactions is provided by invoking the differences in isoscalar and isovector contributions.
\end{abstract}

(c) 2007 Elsevier B.V. All rights reserved.

PACS: 25.30.Pt; 25.30.Fj; 24.10.Jv

Extensive analyses of quasielastic (QE) inclusive electron scattering data performed in recent years [1-4] have clearly demonstrated the quality of the behavior known as scaling. These analyses are based on the so-called superscaling function, $f\left(\psi^{\prime}\right)$, obtained by dividing the cross section by an appropriate function which contains the single nucleon physics, and plotting the result against the scaling variable $\psi^{\prime}(q, \omega)$ (see, e.g., [5]). One then studies the dependences upon the momentum transfer $q$ and the specific nucleus chosen. From $\left(e, e^{\prime}\right)$ world data one concludes that scaling of the first kind (no dependence on $q$ ) is reasonably respected at energies below the QE peak, whereas scaling of the second kind (no dependence on the nuclear species) is fulfilled very well in the same region. The simultaneous occurrence of both kinds of scaling is called superscaling. At energies above the QE peak, breaking of both

\footnotetext{
* Corresponding author.

E-mail address: jac@us.es (J.A. Caballero).
}

kinds of scaling is observed, residing mostly in the transverse channel, and likely due to effects beyond the impulse approximation.

Experimental data lead to a scaling function with a characteristic asymmetric shape, having a tail that extends to high values of the transferred energy $\omega$ (positive values of the scaling variable $\left.\psi^{\prime}(q, \omega)\right)$. The asymmetric shape of the scaling function is largely absent in non-relativistic (NR) models based on a mean field approach. In contrast, the study presented in $[6,7]$ has shown that the asymmetry can in fact be obtained within the relativistic impulse approximation (RIA), given that a description of final-state interactions (FSI) using strong relativistic mean field (RMF) potentials is assumed. Recently, we have shown [8] that an asymmetrical scaling function can be also obtained within the framework of a semi-relativistic (SR) model, based on improved NR expansions of the on-shell electromagnetic current, provided that the FSI are described by the Dirac equation-based (DEB) potential [9] derived from the RMF. Note that, in the SR model, the nonlocalities arising from the 
NR reduction of the Dirac equation - and incorporated into the wave functions through the Darwin factor-are essential to reproduce the asymmetric shape of the scaling function.

The data analysis of the separated longitudinal $(L)$ and transverse $(T)$ contributions to the scaling function carried out in [3, 4] has shown that, whereas the $L$ response does scale to a universal curve, the $T$ strength increases with the transfer momentum $q$ and/or the mass number $A$. This excess of strength in the transverse channel is not entirely understood, although different effects ranging from FSI effects to MEC contributions have been invoked to explain it. This result also connects with the breaking of the zeroth-kind scaling (defined as the equality of the scaling functions obtained from the separated $L$ and $T$ contributions, viz., $\left.f_{L}\left(\psi^{\prime}\right)=f_{T}\left(\psi^{\prime}\right)=f\left(\psi^{\prime}\right)\right)$ observed in the data. From previous studies $[7,8,10]$ it turned out that the zeroth-kind scaling is closely fulfilled by various models based on the impulse approximation. This is the case of the Relativistic Fermi Gas (RFG), by construction. The use of traditional NR and SR approaches also leads to similar longitudinal and transverse scaling functions. This occurs for different descriptions of the FSI, namely, using the same Woods-Saxon potential as in the initial state, which leads to symmetrical scaling functions that do not agree with experiment [10], and making use of the DEB potential that produces the correct amount of asymmetry in the scaling function [8]. Finally, the RIA also leads to the fulfilment of zeroth-kind scaling when the plane wave limit is assumed [7], i.e., the final nucleon state is described as a free relativistic on-shell particle. This is known as Relativistic Plane Wave Impulse Approximation (RPWIA).

Contrary to the above-mentioned models, breakdown of zeroth-kind scaling is observed in the RIA model with FSI described by the strong scalar and vector potentials of the RMF, which yields a scaling function found to be in excellent agreement with the data. The amount of scaling violation depends on the particular prescription chosen for the current operator. This is illustrated in the upper panels of Fig. 1, where we show $f\left(\psi^{\prime}\right)$ as well as its longitudinal $f_{L}\left(\psi^{\prime}\right)$ and transverse $f_{T}\left(\psi^{\prime}\right)$ contributions for ${ }^{12} \mathrm{C}$. The momentum transfer has been fixed to $q=1 \mathrm{GeV} / c$ when computing $f_{L, T}$, while, for the global scaling function $f$, in addition the beam energy has been fixed to $\varepsilon=1 \mathrm{GeV}$, implying that the scattering angle is a function of $\psi^{\prime}$. The left and right panels correspond to results for the $\mathrm{CC} 2$ and $\mathrm{CC} 1$ prescriptions of the electromagnetic current operator, respectively $[7,11]$. In both cases breaking of zeroth-kind scaling is clearly observed, $f_{T}$ being about $20 \%$ larger than $f_{L}$ for $\mathrm{CC} 2$ and almost twice as large for CC1. This result, first observed in [7], differs from the RPWIA and SR models considered in the literature $[7,8,10]$ where zeroth-kind scaling is well obeyed. Moreover, it turns out in the so-called scaling region $\left(\psi^{\prime}<0\right)$ that, within the RMF model, scaling of the first kind is fulfilled separately by $f_{L}$ and $f_{T}$ with only modest scaling violations in each case. However, the two scaling functions obtained are different, with $f_{T}$ lying higher than $f_{L}$.

We should point out once more that, in contrast, the SR approach with a convenient description of FSI based on the DEB potential (derived from the same relativistic Hartree potential) yields results [8] consistent with the asymmetry shown by the
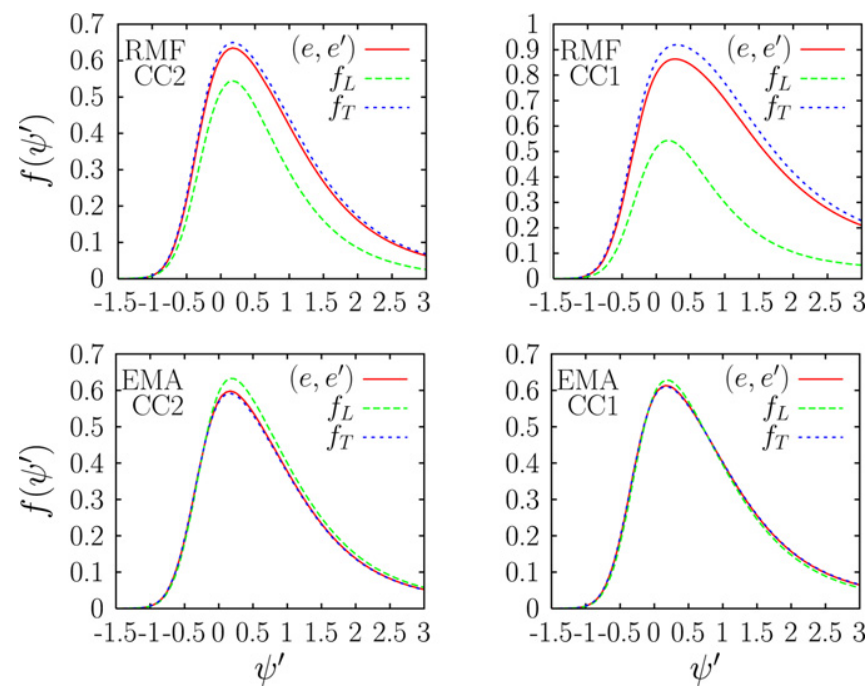

Fig. 1. (Color online.) Scaling function $f\left(\psi^{\prime}\right)$ compared with separate $L$ and $T$ contributions. All results correspond to the analysis of $\mathrm{QE}\left(e, e^{\prime}\right)$ on ${ }^{12} \mathrm{C}$. For the separate scaling functions the momentum transfer is $q=1 \mathrm{GeV} / c$, while for the $\left(e, e^{\prime}\right)$ results the incident electron energy is $\varepsilon=1 \mathrm{GeV}$. The results correspond to the RMF description of FSI (top panels) and the EMA approach (bottom). The current operators CC2 (left) and CC1 (right) have been considered.

RMF model and data, but which respect zeroth-kind scaling almost exactly. Although extensive tests showing the reliability of the SR expansion [12] have been performed within the context of the RFG and RPWIA approaches, the effects introduced by the dynamical enhancement of the lower components in the Dirac spinors [13-15], accounted for within a fully relativistic calculation, are not present in the SR approaches and this may be the reason that the fully relativistic and the SR calculations for the scaling function differ. From previous studies [6,7], we have shown that the scaling function is very slightly modified by the dynamical relativistic effects linked to the initial bound nucleon states.

The presence of strong scalar and vector potentials in the nuclear states (mainly the final one) leads to a significant enhancement of the lower components of the four-spinors describing the nucleon wave functions in the relativistic approach. Due to this, the ratio between upper and lower components of the fully relativistic calculation is quite different from the one for free spinors or implied in the NR or SR approaches [1517]. In order to assess the influence of the enhancement of the lower components (also called spinor distortion [18]) the fully relativistic calculation is compared here with the effective momentum approach (EMA) $[9,15,17,18]$. In the EMA the relationship between upper and lower components is forced to be the same as for free spinors. Thus the EMA wave functions lack the dynamical enhancement of the lower components due to the presence of strong potentials. This is also the case of SR approaches where projections over positive-energy states and truncated expressions of the current operator are considered. Lacking this kind of distortion, the EMA results should lie closer to the so-called factorized result $[15,17]$.

Scaling of the zeroth kind is well fullfilled in the EMA model, as is clearly illustrated in the bottom panels of Fig. 1, 
where we present the scaling functions again corresponding to the two currents operators, now evaluated with the EMA. These results illustrate the crucial role played by spinor distortion in leading to zeroth-kind scaling violations. In fact, the EMA approach leads to $f\left(\psi^{\prime}\right) \approx f_{L}\left(\psi^{\prime}\right) \approx f_{T}\left(\psi^{\prime}\right)$ using either of the current operators.

This result is in accordance with the study performed in the context of the SR approach and the DEB description of FSI [8]. To conclude, notice that projecting out the negativeenergy components yields an enhancement (decrease) of the $L$ $(T)$ contributions. The magnitude of these effects being moderate (strong) for $\mathrm{CC} 2$ (CC1) current operators. This is in consonance with the known tendency of the $\mathrm{CC} 1$ operator to enhance the effects of spinor distortion in the electromagnetic observables, while $\mathrm{CC} 2$ shows a more moderate dependence on the amount of distortion [13,16,17].

One important application of superscaling has been suggested in [19] within the context of making realistic predictions for charge-changing (CC) neutrino-nucleus differential cross sections which, for example, are of interest in neutrino oscillation experiments. The validity of the superscaling hypothesis, i.e., the existence of a universal scaling function in electroweak processes, constitutes an essential result which is supported by various theoretical studies $[6-8,10]$ and gave rise to recent applications to neutrino studies [20,21]. The universal character of the scaling function is the basis of the SuperScaling Analysis (SuSA) introduced in [19]. Within SuSA, the experimental superscaling function extracted from the analysis of $\left(e, e^{\prime}\right)$ world data is used to reconstruct $\mathrm{CC}$ neutrino-nucleus cross sections. However, it is important to point out that the extraction of the experimental scaling function refers only to the analysis of the longitudinal function, $f_{L}$, whereas, in contrast, $(\nu, \mu)$ reactions are totally dominated by the purely transverse $T_{V V}+T_{A A}$ and $T_{V A}^{\prime}$ channels. Thus, one may question the validity of using $f_{L}$ extracted from electron scattering data to predict $(\nu, \mu)$ cross sections, which are dominated by transverse responses. This issue is particularly relevant within theoretical frameworks which lead to $f_{L}\left(\psi^{\prime}\right) \neq f_{T}\left(\psi^{\prime}\right)$, i.e., violation of zeroth-kind scaling. In what follows we present a study of this issue within the RMF approach. Our aim is to answer the above question and clarify the degree to which the scaling hypothesis does or does not work.

In Fig. 2 we present the scaling functions obtained from the calculation of CC neutrino and antineutrino-nucleus reaction cross sections. The kinematics correspond to neutrino (antineutrino) energy fixed to $\varepsilon=1 \mathrm{GeV}$ and lepton scattering angle $\theta_{\mu}=45^{\circ}$. In each graph we show the results obtained with the RMF approach applied for neutrinos (solid line) and antineutrinos (dashed) compared with EMA (in this case only the curve for neutrinos is presented as the one for antineutrinos is very similar). The separate analysis of the transverse channels, $T$ and $T^{\prime}$, is also shown in middle and bottom panels, respectively. The longitudinal contribution to inclusive CC neutrino-nucleus scattering is negligible and therefore is not shown. The usual relativistic single-nucleon expression for the charged-current operator $[14,19]$ has been employed. From results in Fig. 2, several basic conclusions emerge. First, the scaling functions for
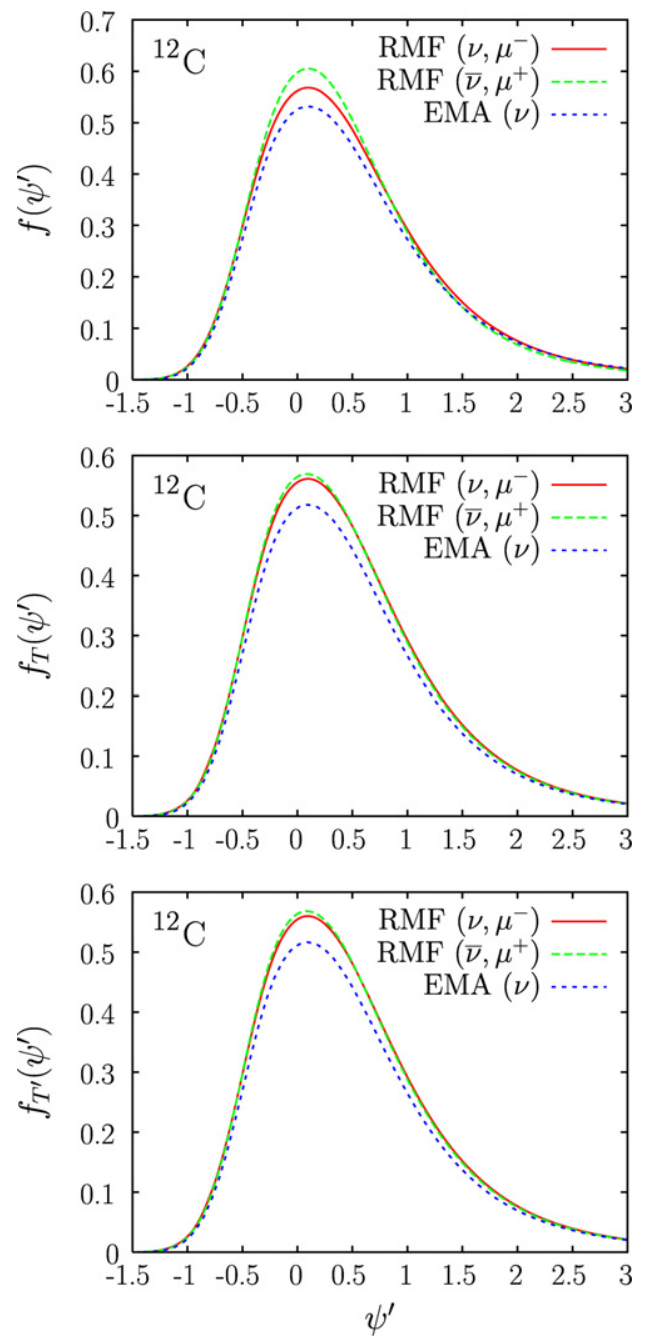

Fig. 2. (Color online.) Scaling functions evaluated from $\left(v, \mu^{-}\right)$and $\left(\bar{v}, \mu^{+}\right)$ reactions. The results correspond to $\varepsilon_{v(\bar{v})}=1 \mathrm{GeV}$ and $\theta_{\mu}=45^{\circ}$. Results from relativistic calculations using the RMF are compared with EMA (see text for details). Top, middle and bottom panels refer to the global scaling function, the $T_{V V}+T_{A A}$ contribution and the $T_{V A}^{\prime}$ one, respectively.

both processes $\left(\nu, \mu^{-}\right)$and $\left(\bar{v}, \mu^{+}\right)$almost coincide. Second, it is verified that $f\left(\psi^{\prime}\right) \approx f_{T}\left(\psi^{\prime}\right) \approx f_{T^{\prime}}\left(\psi^{\prime}\right)$ to a high degree, i.e., the separate transverse responses contribute similarly to the global scaling function. Notice that this is valid, not only for the EMA approach, but also within the fully RMF model. Third, the dynamical enhancement of the lower components in the Dirac wave functions leads only to a slight modification of the scaling functions. These results are in accord with ones observed for $\left(e, e^{\prime}\right)$ reactions in the $\mathrm{CC} 2$ case (see Fig. 1).

For the same kinematics, the basic difference between electron and neutrino scattering is the nature of the exchanged vector boson, a virtual photon probing the electromagnetic current in electron scattering, and a $W^{ \pm}$probing the weak current in CC neutrino-nucleus processes. As a consequence, whereas for inclusive $\left(e, e^{\prime}\right)$ processes all nucleons (protons and neutrons) in the nucleus contribute, in the case of CC neutrino (antineutrino) reactions only the neutrons (protons) in the target contribute to the inclusive cross section. 

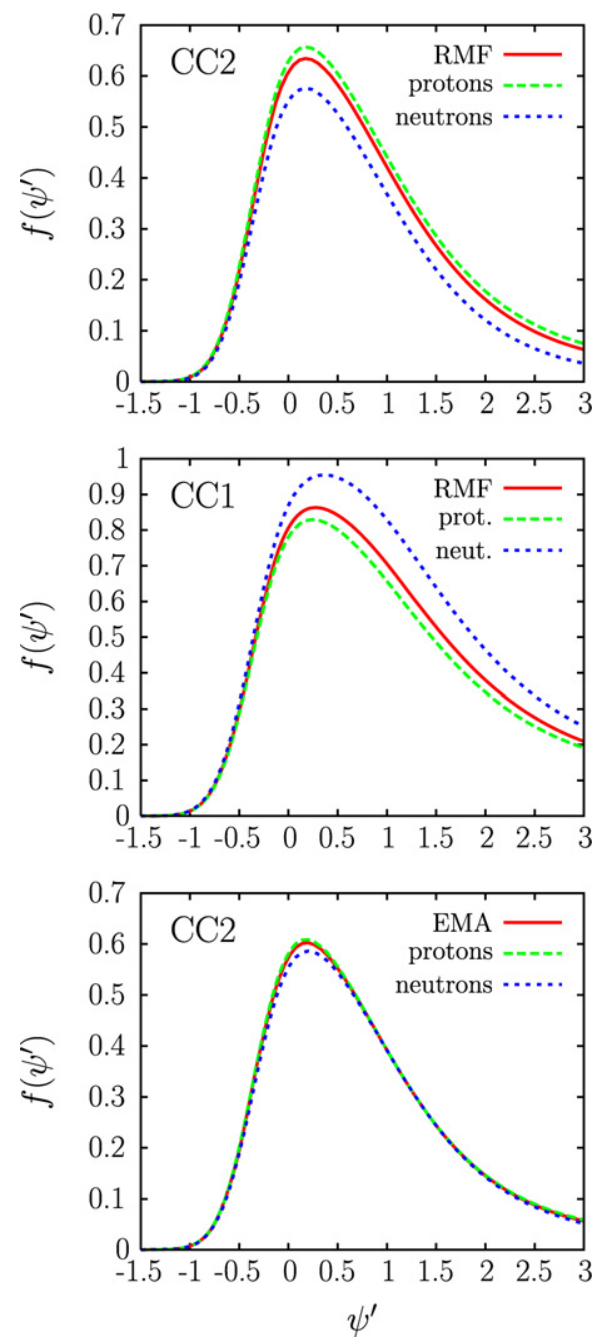

Fig. 3. (Color online.) Proton and neutron contributions to the scaling function obtained from the analysis of ${ }^{12} \mathrm{C}\left(e, e^{\prime}\right)$. Same kinematics as in Fig. 1. The results correspond to the RMF approach with $\mathrm{CC} 2$ (top panel) and CC1 (middle). The EMA case is presented in the bottom panel.

In order to compare $\left(e, e^{\prime}\right)$ and $\left(v, \mu^{-}\right)$reactions, it is convenient to separate the contributions of protons and neutrons to the $\left(e, e^{\prime}\right)$ scaling function in the form

$f\left(\psi^{\prime}\right)=\frac{G_{P}}{G} f_{P}\left(\psi^{\prime}\right)+\frac{G_{N}}{G} f_{N}\left(\psi^{\prime}\right)$,

where $G_{P}$ and $G_{N}$ are the single-nucleon functions (incorporating both the longitudinal $L$ and transverse $T$ contributions) defined in $[4,7]$ for protons and neutrons, respectively, and $G=G_{P}+G_{N}$. The proton $f_{P}$ and neutron $f_{N}$ scaling functions are shown in Fig. 3 for the same kinematics as in Fig. 1. The total scaling function, obtained as an average of $f_{P}$ and $f_{N}$ with weights $G_{P} / G$ and $G_{N} / G$, respectively, is also shown in the figure with solid lines. We show results for the RMF approach and for both current operators CC2 (top panel) and CC1 (middle). For completeness, the results corresponding to EMA are also shown in the bottom panel (here we only consider CC2, since results for $\mathrm{CC} 1$ are basically the same). As one observes, in EMA the proton and neutron scaling functions are almost identical, i.e., $f_{P}=f_{N}$; such a result will be called scaling of

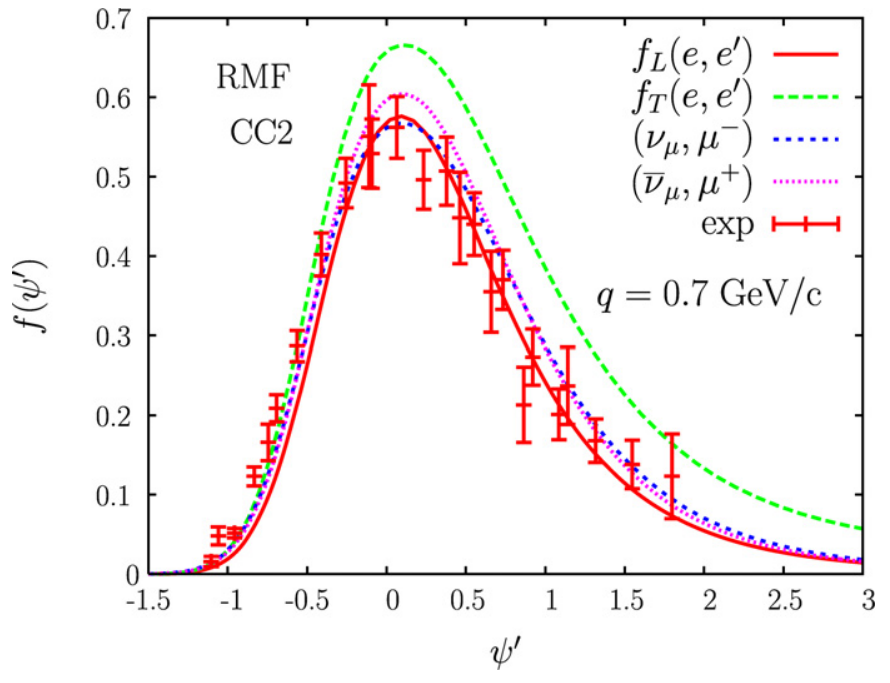

Fig. 4. (Color online.) Longitudinal and transverse scaling functions for $\left(e, e^{\prime}\right)$ compared with $f\left(\psi^{\prime}\right)$ evaluated from $\left(\nu, \mu^{-}\right)$and $\left(\bar{v}, \mu^{+}\right)$. All results have been evaluated with the RMF approach using the $\mathrm{CC} 2$ current operator. The kinematics selected correspond to fixed values of the incident lepton energy, $\varepsilon=1 \mathrm{GeV}$, and momentum transfer, $q=0.7 \mathrm{GeV} / c$. The averaged experimental function extracted from longitudinal electron scattering data is also shown [4].

the third kind or isospin scaling. A similar result also occurs for the NR and SR calculations. In contrast, protons and neutrons yield different scaling functions in the RMF approach, with magnitude dependent on the choice of current operator. The results of Fig. 3 indicate that the proton/neutron balance in $f\left(\psi^{\prime}\right)$ is significantly modified after the inclusion of dynamical relativistic distortion of the spinors, due mainly to the presence of strong relativistic potentials in the final state. Thus, the effect of spinor distortion on the scaling function is isospin-dependent, making the separate scaling functions for protons and neutrons appreciably different, $f_{P} \neq f_{N}$, that is, violating scaling of the third kind. This is connected to the breaking of the zeroth-kind scaling observed in Fig. 1 in the full relativistic calculation. As will be shown later, the isoscalar/isovector terms in both the $L$ and $T$ channels in $\left(e, e^{\prime}\right)$ reactions also yield significant differences when compared with the purely isovector contributions involved in CC neutrino-nucleus scattering processes.

The universal character of the scaling function and its validity for electromagnetic and weak interactions is further analyzed in Fig. 4. Here we directly compare the functions $f_{L}\left(\psi^{\prime}\right)$ and $f_{T}\left(\psi^{\prime}\right)$ obtained from $\left(e, e^{\prime}\right)$ cross sections with the ones corresponding to $\left(v_{\mu}, \mu^{-}\right)$and $\left(\bar{v}_{\mu}, \mu^{+}\right)$reactions. All results have been evaluated within the RIA and making use of the RMF potential to describe FSI. The prescription of the current operator is $\mathrm{CC} 2$ for both electromagnetic and weak interactions [7]. The kinematics correspond to fixed values of the incident lepton (electron, neutrino, antineutrino) energies, $1 \mathrm{GeV}$, and transferred momentum $q=0.7 \mathrm{GeV} / c$. Similar results are obtained if, instead of fixing the momentum transfer, we select a specific value of the lepton (electron or muon) scattering angle. The averaged QE phenomenological function obtained from the analysis of $\left(e, e^{\prime}\right)$ data [4] is also included in Fig. 4. As observed, the theoretical curve for $f_{L}\left(\psi^{\prime}\right)$ follows the behavior 
of the data very closely (referred only to the analysis of the longitudinal scaling function), and this proves the capability of the RIA combined with the RMF potential to describe $\left(e, e^{\prime}\right)$ data in the longitudinal channel satisfactorily. On the contrary, the transverse contribution $f_{T}\left(\psi^{\prime}\right)$ overestimates the data by $\sim 20 \%$ even in the region close to the maximum, $\psi^{\prime} \approx 0$. This result, arising from zeroth-kind scaling violation in the RMF approach, is not in conflict with $\left(e, e^{\prime}\right)$ data that indeed leaves room for effects of this type (see the general discussion in $[3,4])$.

Concerning the scaling function obtained for neutrino (and antineutrino) scattering reactions, one observes that it is much more in accordance with $f_{L}\left(\psi^{\prime}\right)$ and hence with the electron scattering longitudinal data, than with $f_{T}\left(\psi^{\prime}\right)$. Within the context of our model this outcome reinforces the validity of the general assumption implied by SuSA [19], i.e., the use of the phenomenological scaling function (extracted from the analysis of longitudinal $\mathrm{QE}$ electron scattering data) to predict $\mathrm{CC}$ neutrino-nucleus cross sections. However, it is also striking that $f\left(\psi^{\prime}\right)$ for $v_{\mu}$ and $\bar{v}_{\mu}$ reactions, which are totally dominated by the purely transverse $\left(T, T^{\prime}\right)$ channels, coincides with the $f_{L}$ function of $\left(e, e^{\prime}\right)$ instead of $f_{T}$, in contrast to what one might expect.

In order to understand these results, let us start by discussing some basic differences between $\left(e, e^{\prime}\right)$ and $(\nu, \mu)$ reactions. In the former, the longitudinal and transverse channels contribute importantly (at least for some kinematics), and in both responses isoscalar and isovector form factors enter. However, as shown in Fig. 3, the balance between isovector and isoscalar contributions may change in a significant way due to the strong dynamical enhancement of the lower components in the outgoing nucleon Dirac wave functions obtained with the RMF potential. In contrast, only purely isovector form factors enter in $\mathrm{CC}$ neutrino-nucleus scattering. Hence, it is important to evaluate isovector and isoscalar contributions in $\left(e, e^{\prime}\right)$ reactions and their effects on the scaling functions. In what follows we investigate how the functions $f_{L}$ and $f_{T}$ obtained from $\left(e, e^{\prime}\right)$ RMF calculations change when the isoscalar form factors are removed. Notice that proceeding in this way, we force the $\left(e, e^{\prime}\right)$ to be purely isovector, similar to what occurs for $(\nu, \mu)$.

The results of our analysis are presented in Fig. 5 for the same kinematics as in Fig. 4. Again, we compare the scaling functions $f_{L}$ and $f_{T}$ for electrons with those of neutrinos and antineutrinos. Experimental $\left(e, e^{\prime}\right)$ data are also included for reference. Top and bottom panels refer to different assumptions concerning the electromagnetic form factors entering in $\left(e, e^{\prime}\right)$ reactions. First, the curves $f_{L}$ and $f_{T}$ in the top panel have been obtained assuming $G_{M}^{n}=-G_{M}^{p}$, i.e., the proton and neutron magnetic form factors are simply set equal in size and opposite in sign. In this way, we remove the isoscalar contribution in the magnetic form factor which, consequently, becomes purely isovector. The proton and neutron electric form factors are not modified. Therefore, results for $\left(e, e^{\prime}\right)$ in the top panel of Fig. 5 reflect the scaling functions where the isoscalar contribution only enters through the electric content of the nucleons. It is important to point out that the strength of the transverse nuclear response function $R^{T}$ increases significantly when isoscalar

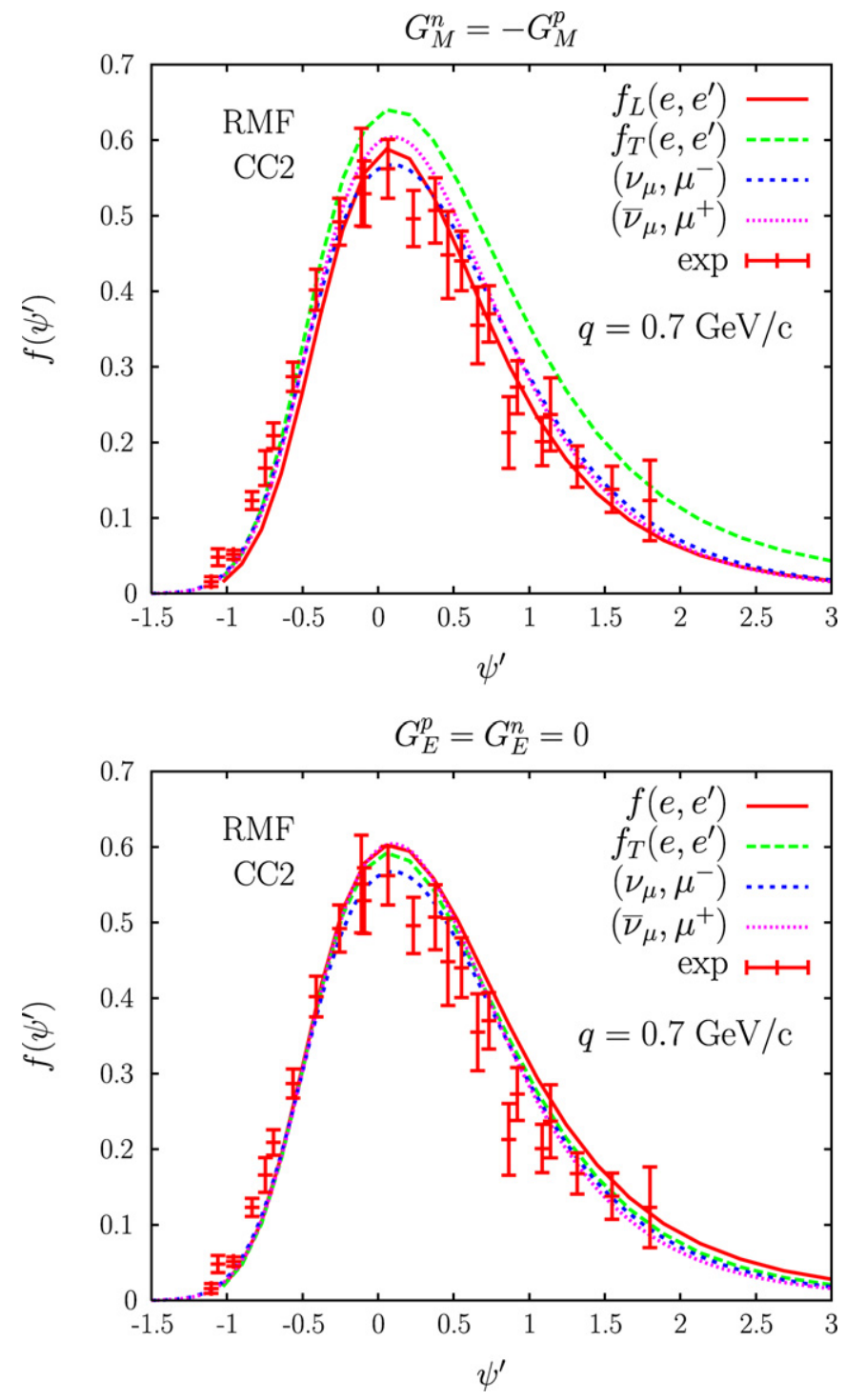

Fig. 5. (Color online.) Same as Fig. 4, but with modified isoscalar/isovector contributions via the nucleon form factors in the $\left(e, e^{\prime}\right)$ case. Top panel: $G_{M}^{n}=-G_{M}^{p}$, i.e., magnetic form factor purely isovector. Bottom panel: $G_{E}^{n}=G_{E}^{p}=0$, i.e., no convective terms.

contributions in the magnetic form factor are removed. However, concerning the scaling functions, the results in Fig. 5, when compared with Fig. 4, show that the discrepancy between $f_{L}$ and $f_{T}$ gets smaller because a visible decrease occurs for $f_{T}$. In other words, removing the isoscalar contribution in $G_{M}$ leads to a weaker violation of the zeroth-kind scaling property (within the RMF context).

In the bottom panel of Fig. 5 we show the results corresponding to no convective terms, i.e., the electric form factors for protons and neutrons (in the electromagnetic sector) are forced to be zero. This is a very drastic assumption which leads to nuclear electromagnetic longitudinal responses $R^{L}$ being very close to zero, having only relativistic-order contributions involving $G_{M}^{p}$ or $G_{M}^{n}$. The reason to consider this non-convective limit comes from the effects introduced by the isoscalar/isovector contributions in the electric form factors of the nucleon. $\mathrm{Ob}$ - 
viously, the neglect of convective terms yields neither isoscalar nor isovector contributions. While being aware of the important differences introduced in the cross sections due to the assumption $G_{E}^{p}=G_{E}^{n}=0$, the analysis of scaling functions, constructed by taking the proper ratios between the nuclear responses and the single-nucleon ones, requires the use of the same approach (no isoscalar or no convective terms) in both the numerator (hadronic dependence) and denominator (singlenucleon). Hence, it is instructive to explore the behavior of the scaling functions in such approximations. The results in the bottom panel of Fig. 5 show that a unique (universal) scaling function emerges from the analysis of $\left(e, e^{\prime}\right)$ calculated cross sections. Moreover, this function (without isoscalar terms) agrees with the one evaluated from $\left(v_{\mu}, \mu^{-}\right)$and $\left(\bar{v}_{\mu}, \mu^{+}\right)$processes and with $f_{\exp }\left(\psi^{\prime}\right)$ extracted from the analysis of longitudinal $\left(e, e^{\prime}\right)$ world data.

In conclusion, we have investigated isospin effects in quasielastic electron and CC neutrino-nucleus cross sections making use of the RIA and various descriptions of FSI with special emphasis placed on the RMF approach. Our main results can be summarized as follows:

- The RMF description of FSI leads to a clear violation of zeroth-kind scaling. This violation only occurs in this model, and not in other approaches based on non-relativistic, semi-relativistic or relativistic plane-wave models.

- The breaking of zeroth-kind scaling has been proven to be due to the important dynamical enhancement of the lower components in the Dirac wave functions (mainly in the final state) produced by the use of strong scalar and vector relativistic potentials.

- The balance between proton and neutron contributions in the scaling functions, namely third-kind scaling, evaluated from $\left(e, e^{\prime}\right)$ reactions is also significantly affected by dynamical relativistic effects. This contrasts with NR, SR and RPWIA approaches, where protons and neutrons lead to similar scaling functions. The same comment applies to the effective momentum approach.

- Finally, we have investigated in more depth the origin of the differences observed between the scaling functions occurring in electron scattering and $\mathrm{CC}$ neutrino reactions. Contrary to what intuition would suggest, the longitudinal scaling function for electron scattering is found to agree with the neutrino-scattering $f$ (which is purely transverse) much better than does the transverse scaling function from electron scattering. We have shown that this result is consistent with the different roles played by isoscalar and isovector nucleon form factors in the two processes. In part this result probably arises because the convective effects are less important for the $\mathrm{CC}$ neutrino reaction than for electron scattering, especially for the axial-vector contributions in the former which are dominated by spin-flip matrix elements.

These general results complement other previous findings [6,7, 10] and support the essential assumption of SuSA. Furthermore, the zeroth-kind scaling violations, not present in other models, may give us some clues as to how to proceed when trying to disentangle the separate roles played by isoscalar and isovector form factors. Obviously, more precision data, particularly for separated longitudinal and transverse contributions to the cross sections and their effects on the scaling functions, are needed. The present experimental results indicate an excess of transverse strength below pion production threshold. It is important to identify the source of this strength (FSI effects in $L$ and $T$ channels, relativistic dynamics, effects beyond the impulse approximation...). The present study should be considered as a step in this direction.

\section{Acknowledgements}

This work was partially supported by DGI (Spain) and FEDER funds under Contracts Nos. FIS2005-01105, FIS200500810, FPA2006-13807 and FPA2006-07393, by the Junta de Andalucía, by the Comunidad de Madrid and UCM (project 910059 'Grupo de Física Nuclear'), and by the INFN-CICYT collaboration agreement (project "Study of relativistic dynamics in electron and neutrino scattering"). It was also supported in part (TWD) by the US Department of Energy Office of $\mathrm{Nu}$ clear Physics under contract No. DE-FG02-94ER40818. Part of the computations were performed in the 'high capacity computational cluster for physics' of UCM, funded in part by UCM and in part with FEDER funds.

\section{References}

[1] D.B. Day, J.S. McCarthy, T.W. Donnelly, I. Sick, Annu. Rev. Nucl. Part. Sci. 40 (1990) 357.

[2] T.W. Donnelly, I. Sick, Phys. Rev. Lett. 82 (1999) 3212.

[3] T.W. Donnelly, I. Sick, Phys. Rev. C 60 (1999) 065502.

[4] C. Maieron, T.W. Donnelly, I. Sick, Phys. Rev. C 65 (2002) 025502.

[5] M.B. Barbaro, R. Cenni, A. De Pace, T.W. Donnelly, A. Molinari, Nucl. Phys. A 643 (1998) 137.

[6] J.A. Caballero, J.E. Amaro, M.B. Barbaro, T.W. Donnelly, C. Maieron, J.M. Udías, Phys. Rev. Lett. 95 (2005) 252502.

[7] J.A. Caballero, Phys. Rev. C 74 (2006) 015502.

[8] J.E. Amaro, M.B. Barbaro, J.A. Caballero, T.W. Donnelly, J.M. Udías, Phys. Rev. C 75 (2007) 034613.

[9] J.M. Udías, et al., Phys. Rev. C 48 (1993) 2731; J.M. Udías, et al., Phys. Rev. C 51 (1995) 3246; J.M. Udías, et al., Phys. Rev. C 64 (2001) 024614.

[10] J.E. Amaro, M.B. Barbaro, J.A. Caballero, T.W. Donnelly, C. Maieron, Phys. Rev. C 71 (2005) 065501.

[11] T. de Forest, Nucl. Phys. A 392 (1983) 232.

[12] J.E. Amaro, J.A. Caballero, T.W. Donnelly, E. Moya de Guerra, A.M. Lallena, J.M. Udías, Nucl. Phys. A 602 (1996) 263;

S. Jeschonnek, T.W. Donnelly, Phys. Rev. C 57 (1998) 2438;

J.E. Amaro, M.B. Barbaro, J.A. Caballero, T.W. Donnelly, A. Molinari, Phys. Rep. 368 (2002) 317.

[13] J.A. Caballero, T.W. Donnelly, E. Moya de Guerra, J.M. Udias, Nucl. Phys. A 643 (1998) 189;

J.A. Caballero, T.W. Donnelly, E. Moya de Guerra, J.M. Udias, Nucl. Phys. A 632 (1998) 323.

[14] M.C. Martínez, J. Vignote, J.A. Caballero, T.W. Donnelly, E. Moya de Guerra, J.M. Udías, Phys. Rev. C 69 (2004) 034604.

[15] J.M. Udias, J.R. Vignote, E. Moya de Guerra, A. Escuderos, J.A. Caballero, in: Procedings 5th Workshop on Electromagnetically Induced Two Hadron Emission, Lund, Sweden, June 2001, nucl-th/0109077.

[16] J.M. Udias, J.A. Caballero, E. Moya de Guerra, J.E. Amaro, T.W. Donnelly, Phys. Rev. Lett. 83 (1999) 5451. 
[17] J.R. Vignote, M.C. Martinez, J.A. Caballero, E.M. de Guerra, J.M. Udias, Phys. Rev. C 70 (2004) 044608.

[18] J.J. Kelly, Phys. Rev. C 56 (1997) 2672;

J.J. Kelly, Phys. Rev. C 59 (1999) 3256.

[19] J.E. Amaro, M.B. Barbaro, J.A. Caballero, T.W. Donnelly, A. Molinari, I. Sick, Phys. Rev. C 71 (2005) 015501.
[20] A.N. Antonov, et al., Phys. Rev. C 74 (2006) 054603.

[21] M. Martini, G. Co', M. Anguiano, A.M. Lallena, Phys. Rev. C 75 (2007) 034604. 